AC 2012-3002: PATENT CLASSIFICATION REFORM: IMPLICATIONS

FOR TEACHING, LEARNING, AND USING THE PATENT LITERATURE

Mr. Michael J. White, Queen's University

(C)American Society for Engineering Education, 2012

$\mathbb{D}$
0
0
$N$
0
$\overrightarrow{0}$
$N$
0 


\section{Patent Classification Reform: Implications for Teaching, Learning and Using the Patent Literature}

\section{Introduction}

In early 2011 the European Patent Office (EPO) and U.S. Patent and Trademark Office (USPTO) announced a project to create a joint classification system called the Cooperative Patent Classification (CPC). The CPC will be based on the European Classification (ECLA) and the International Patent Classification (IPC) and incorporate the best classification practices from the USPTO. Starting in 2013 the EPO and USPTO will classify all EP and US documents using the CPC. This development has significant implications for academic engineering librarians. Patents are one of the core literatures of engineering and have been taught in engineering schools for more than a century. As early as 1913, engineering students at Cornell University attended lectures on patent topics. ${ }^{1}$ Today, academic engineering librarians often provide formal and informal instruction to students on how to search the patent literature, including how to use patent classification codes to retrieve patents related to specific technologies. In an informal survey of academic engineering librarians conducted by the author in 2008, 51 percent of the respondents reported that patent literature was included in the engineering curriculum at their institutions. Nearly 58 percent also indicated that they teach workshops on patent searching once or more per year. ${ }^{2}$ Numerous engineering libraries in North America offer research guides to the patent literature and tutorials on patent searching.

This paper will explore how the CPC will be implemented in patent databases and its potential impacts on how engineering librarians teach students to use the patent literature. The author will describe the organization of the CPC and its relationship to current classification systems.

\section{Principles of Patent Classification}

Patent classification systems arose from the need to organize, store and retrieve printed patent documents efficiently and quickly. Even today in an era of massive online patent databases, integrated thesauri, and semantic search engines, patent classification is a useful tool for prior art searching and patent analysis. Keyword searches in patent databases are problematic for several reasons. First of all, keyword searches locate words, not ideas or concepts. It is very difficult to include in a search all the terms and synonyms that may represent a technological concept. ${ }^{3}$ Language presents another major challenge to keyword searching. If you search an international patent database such as Espacenet using only English keywords, you will miss non-English documents from China, Korea, Japan, Russia, etc. Patent classification overcomes these obstacles. The major patent classification systems in use today include the International Patent Classification (IPC), the European Classification (ECLA), the Japan Patent Office F-term system, and the U.S. Patent Classification (USPC). This paper will focus mainly on the USPC, ECLA and IPC. 


\section{U.S. Patent Classification System (USPC)}

The current USPC system is based on a scheme first adopted in $1900 .{ }^{4}$ Since its inception, the USPC has grown, somewhat organically, from approximately 200 classes to more than 450 classes. Today the USPC consists of 437 utility classes numbered 2 through 987, 32 design classes numbered D1 through D99, and one class designated PLT for plant patents. Each class has a title that describes the subject matter it covers. For example, Class 280, Land Vehicles includes non-motorized transportation having wheels and/or runners, such as sleighs, sleds, bicycles, skateboards, scooters, wheelbarrows, peddle carts, etc. Motorized vehicles, on the other hand, are classified in Class 180. USPC codes first appeared in patent abstracts in the Official Gazette of the U.S. Patent Office around 1920 and on issued patents from August 1933 forward. (See US 1,920,165.) USPC codes are included in many public and commercial patent databases.

Although the USPC system is based on hierarchical principles, it actually has a relatively flat structure. The top level consists of about 470 classes, while the second level consists of approximately 150,000 subclasses. ${ }^{5}$ A USPC code, therefore, consists of two parts, a class followed by a subclass. For example, $280 / 287$ is the code for subject matter relating to folding or collapsible bicycles, tricycles, scooters, etc. The hierarchical level among subclasses is indicated by dots and the placement of subclasses within a schedule. (See Figure 1.) This dual-level structure is also found in design and plant classes. For example, D12/111 is the design code for bicycles designs, including foldable bicycle frames.

Figure 1. USPC Schedule (Collapsed) for Folding Bicycles.

$\begin{array}{ll}\text { CLASS } & \text { 280, LAND VEHICLES } \\ 29 & \text { WHEELED } \\ 200 & \text {. Occupant propelled type } \\ 263 & \text {.. With steering } \\ 270 & \text {... One-wheel controlled } \\ 274 & \text {.... Frames and running gear } \\ 278 & \text {..... Extensible and knockdown }\end{array}$

There is no fixed relationship between class or subclass numbers and technological subject matter. It is not uncommon to find very different subject matter assigned to proximate classes. For example, consider the following class series:

- Class 257, Active solid-state devices (e.g., transistors, solid-state diodes)

- Class 258, Railway mail delivery

- Class 259, No title (unassigned)

- Class 260, Chemistry of carbon compounds

- Class 261, Gas and liquid contact apparatus 
Obviously, there is little relationship between transistors, railway mail delivery, organic chemistry, and carburetors. The fact that these very different technological fields (electronics, mechanics and chemistry) are in close proximity in the USPC is a product of its organic evolution. The opposite is also true: related subject matter can be found in classes that are far apart, numerically speaking. For example, Class 12, Boot and Shoe Making and Class 36, Boots, Shoes and Leggings are closely related but not proximate in the USPC. Class 259 in the series above is currently vacant and does not appear in the list of classes on the USPC website. There are many unused class numbers in the USPC because of ongoing class reorganization, creation and destruction. Class numbers are recycled. For example, Class 257 was formerly used for heat exchangers.

One way to visualize the unique structure of the USPC is to imagine a train with 1,000 cars, with each car representing a single utility, design or plant class. (Figure 2.) Within each car are dozens or hundreds of boxes representing subclasses. The order of the cars (classes) and the arrangement of the boxes (subclasses) within the cars depend on the theories of classification that existed when the class was last revised. Class numbers 900-999 are reserved for cross-reference art classes, hybrid collections of subject matter drawn from several classes that are grouped together in order to facilitate searching. An example of a cross-reference class is Class 901, Robots. Design and plant classes are located after the utility classes.

Figure 2. Representation of a section of the USPC with classes covering electrical (257), mechanical (258) and chemical (260 and 261) subject matter. Class 259 is unused.
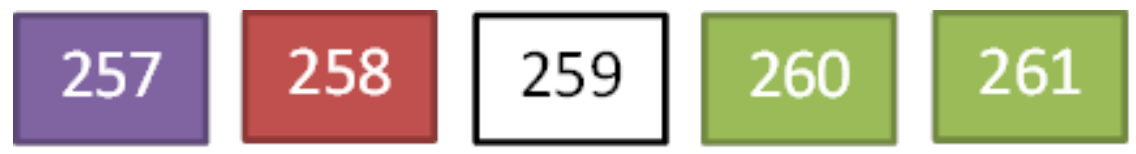

Since its creation, the USPC has evolved to accommodate the growing volume of patent and non-patent literature and to incorporate new technologies. When the number of patents in a class because so large that it inhibits effective searching, the USPTO reorganizes it. Reorganization can consist of moving, renaming, abolishing and/or adding subclasses within a class. When the volume of prior art becomes too large to fit in a single class, the class may be expanded into two or more classes. For example, subject matter related to surgery and surgical instruments is covered in Class 128 (the original class) and classes 600, 601, 602, 604, 606 and 607. Changes to the USPC are announced in classification orders, almost 2,000 of which have been published since 1947. The USPTO updates USPC data in its public databases approximately bimonthly; current USPC codes are searchable but not the historical codes.

The USPTO provides many tools that support the USPC. The Index to the USPC is an alphabetical list of common and technical terms and trade names along with numerical references to a class or subclass. A Consolidate Glossary of U.S. Patent Classification Terms is also available on the USPTO website. This is a comprehensive list of terms extracted from USPC definitions, which are useful to patent searchers because many terms in the USPC have 
technology-specific definitions that are different from standard dictionary definitions. Concordances of USPC-to-IPC codes and IPC-to-USPC codes facilitate translation between the two systems. Numerous guides and tutorials on how to search USPC codes are available on the internet. $^{6-8}$

\section{International Patent Classification (IPC)}

The IPC originated in the 1950s from the collaborative efforts of several European patent offices to create a shared or common patent classification system. Up until that time most patent offices classified patents and non-patent literature under national patent classification systems. As the volume of patent and non-patent literature exploded after the Second World War, this practice became increasingly duplicative, expensive and unsustainable. After further development and testing by patent offices during the 1960s, the IPC was formally established in 1971 under the Strasbourg Agreement. The IPC is governed by the 61 countries that are members of the treaty and administered by the World Intellectual Property Organization. Today, the IPC is used by more than 100 countries worldwide. In addition, IPC codes have been applied to some nonpatent literature databases. For example, IPC codes have been added recently to Inspec, a scientific and technical literature database published by the Institution of Engineering and Technology (IET). ${ }^{9}$

The IPC is a hierarchical classification consisting of five levels. The top level consists of eight Sections, which are further subdivided into classes, subclasses, main groups and sub-groups. The IPC Sections are:

- A Human Necessities

- B Performing Operations; Transporting

- C Chemistry; Metallurgy

- D Textiles; Paper

- E Fixed Constructions

- F Mechanical Engineering; Lighting; Heating; Weapons; Blasting

- G Physics

- H Electricity

The IPC contains about 70,000 individual subgroups, about half the number of ECLA and the USPC. The IPC is updated annually.

Figure 2. IPC Schedule (Collapsed) for Folding Bicycles.

\begin{tabular}{|ll|}
\hline B & PERFORMING OPERATIONS; TRANSPORTING \\
B62 & Land vehicles for travelling otherwise than on rails \\
B62K & Cycles, cycle frames, etc. \\
B62K15/00 & Collapsible or foldable cycles \\
\hline
\end{tabular}




\section{European Classification System (ECLA)}

The European Classification System (ECLA) is based on the IPC and very similar in structure. The top level consists of the same eight sections found in the IPC, plus an additional section Y for new technological developments and cross-reference collections that span several IPC sections. The first hybrid section, Y02, covers technologies and applications related to the mitigation or adaptation against climate change. ECLA has approximately 135,000 individual codes, placing it on part with the USPC. Figure 3 below shows the hierarchy of ECLA codes for folding bicycle frames. Note that the first four codes are the same as in the IPC schedule. The two final codes are ECLA alphanumeric extensions.

Figure 3. ECLA Schedule (Collapsed) for Folding Bicycles.

\begin{tabular}{|ll|}
\hline B & PERFORMING OPERATIONS; TRANSPORTING \\
B62 & Land vehicles for travelling otherwise than on rails \\
B62K & Cycles, cycle frames, etc. \\
B62K15/00 & Collapsible or foldable cycles \\
B62K15/00F & . The frame being foldable \\
B62K15/00F2 & .. Frame being foldable about 2 or more axis \\
\hline
\end{tabular}

The EPO's Espacenet system is the only public patent database that offers searchable ECLA codes. Approximately half of the 70 million patent documents in Espacenet are assigned ECLA codes. This includes U.S. patent documents from 1920 forward. Some countries are only selectively classified. For example, approximately 2.4 million out of 13.6 million Japanese patent documents in Espacenet have ECLA codes. ECLA codes are also applied to most of the two million non-patent literature records in Espacenet. The EPO updates ECLA codes on an ongoing basis and publishes a monthly report that lists codes added, deleted and updated.

\section{Cooperative Patent Classification System (CPC)}

According to EPO and USPTO announcements, the CPC will be based on ECLA and incorporate the best classification practices of both offices. ${ }^{10}$ The CPC will be definitions for each technical field covered by the CPC. These definitions will be used internally by the USPTO and EPO and then made available to the public at a later date. The USPC will be frozen in 2011 and gradually phased out during 2012. The EPO and UPSTO will continue to jointly manage and develop the CPC. By early 2013, both the EPO and USPTO will classify all patent documents under CPC. However, the USPTO will continue to classify design and plant patents under the legacy USPC system.

The CPC will look remarkably similar to ECLA, except that the alphanumeric extensions will be replaced by numbers. ECLA is expected to grow from about 140,000 to 200,000 codes. For example, the code for bicycles with folding frames will change from B62K15/00F in ECLA to B62K15/009 in CPC. 
Figure 4. CPC Schedule (Collapsed) for Folding Bicycles.

\begin{tabular}{|ll|}
\hline B & PERFORMING OPERATIONS; TRANSPORTING \\
B62 & Land vehicles for travelling otherwise than on rails \\
B62K & Cycles, cycle frames, etc. \\
B62K15/00 & Collapsible or foldable cycles \\
B62K15/009 & . The frame being foldable \\
B62K15/0018 & .. Frame being foldable about 2 or more axis \\
\hline
\end{tabular}

\section{CPC Advantages and Disadvantages}

There are numerous benefits, and some disadvantages, arising from the CPC project. First of all, the CPC will be built on the foundations of an already established and continually updated patent classification system, ECLA. By pooling their patent classification expertise, the EPO and USPTO will reduce duplication and improve efficiency within their respective offices. Classification accuracy should also improve as USPTO and EPO examiners develop a better common understanding of classification practices. Most importantly, patent searchers will no longer have to use two classification systems (USPC and ECLA/IPC) in order to do a comprehensive patent search.

The disadvantages of this project primarily concern the USPC. The USPC reflects more than a century of knowledge and experience of USPTO patent examiners. Its organic nature has led to the creation of hundreds of unique codes that might be lost in the migration to the CPC. U.S. documents prior to 1920 will be reclassified in CPC. Thousands of U.S. patent examiners and patent information professional will have to learn a new classification system. However, the similarity between the CPC and IPC/ECLA should not make this too onerous. Patent database producers will have to update their systems to incorporate the new CPC data. The migration to CPC will also require libraries to update their patent guides and create new tutorials explaining how to search the CPC.

\section{Future Developments}

As of March 2012, much is still unknown about the implementation of the CPC. For example, it is expected that the CPC will be revised several times per year, but the exact schedule is yet to be determined. According to the CPC website, the new launch date of the CPC is January 1, 2013. The fate of the USPC is also unclear. The USPC was to have been frozen in November 2010, but revisions to the USPC, published in classification orders, continue to be made. The most recent classification order, No. 1917, Class 351, was published on March 6, 2012. It is not known when or if the USPTO will cease classification of U.S. patents under the USPC or if historical USPC codes will continue to be searchable in USPTO patent databases. 
Figure 5. Folding Bicycle Patent (US 1,584,568). ${ }^{11}$

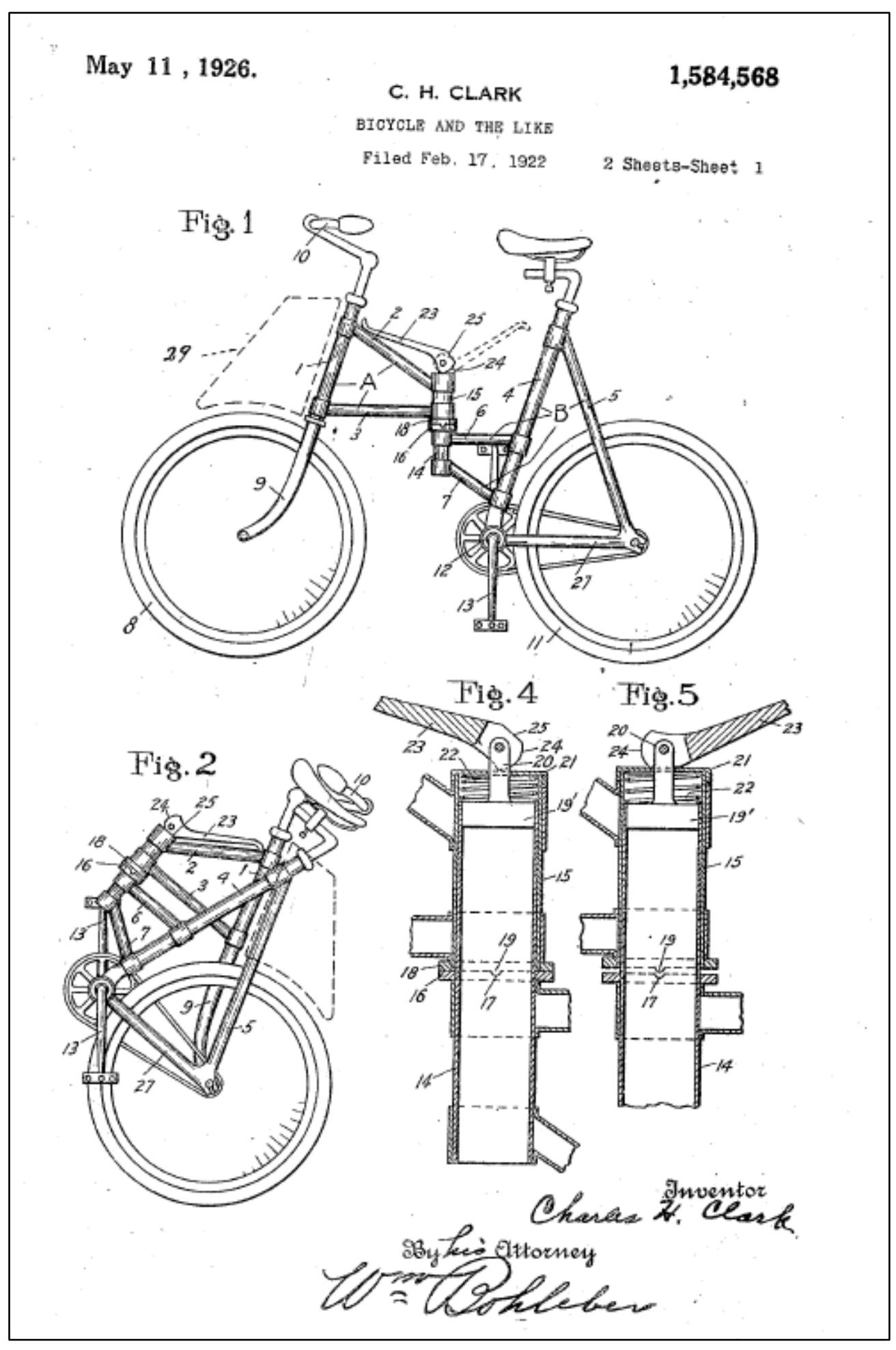

0
$\mathbb{0}$
0
0
0
$\overrightarrow{0}$
$\overrightarrow{0}$
$\infty$
$\infty$ 


\section{Patent Classification Resources}

\section{Cooperative Patent Classification (CPC)}

The CPC website is the best source of news and information about the CPC project. Current documentation includes a presentation on the CPC project. The website URL is

http://www.cooperativepatentclassification.org.

\section{European Classification (ECLA)}

The current version of ECLA can be searched in Espacenet at http://www.epo.org/searching/essentials/classification/ecla.html. Monthly update reports, which are available at http://www.epo.org/searching/essentials/classification/ecla/changes.html, provide additions, deletions and changes to ECLA.

\section{International Patent Classification (IPC)}

The current edition of the IPC is available on the WIPO website at http://www.wipo.org .

\section{U.S. Patent Classification (USPC)}

The USPC is available at http://www.uspto.gov/web/patents/classification. USPC resources include the Index to the USPC System, class schedules and definitions, USPC-IPC8/IPC-USPC concordances, classification orders from 2004 forward, classification orders index, classes under reclass reports, and a consolidated glossary of USPC terms. The USPC index, schedules, definitions and concordances may be downloaded in PDF format.

\section{European Patent Office Online Training}

The EPO offers numerous free online training materials, lectures and podcasts at http://www.epo.org/learning-events/e-learning.html.

\section{Intellogist}

The Intellogist is a free resource for patent information professionals hosted by Landon IP, Inc. It contains patent search system reports, best practices in patent searching, a glossary, discussion forum and a patent coverage map for determining which search systems cover a selected country. The URL is http://www.intellogist.com.

\section{Patent Information News}

Patent Information News is a free newsletter published quarterly by the European Patent Office that often contains helpful tips and information on patent searching. Archived copies back to 2004 are available at http://www.epo.org/service-support/publications/patentinformation/news.html.

\section{References}

[1] W. Macomber, Engineers’ Handbook on Patents, Little, Brown \& Co., Boston, 1913. 
[2] M. White. (2008). How are Engineering Librarians Using Patent Literature? A Pilot Survey [Online]. Available: http://hdl.handle.net/1974/1377.

[3] S. Adams. "Patent Searching Without Words: Why Do It? How to do It?", Free Pint Newsletter, 130, Feb. 6, 2003. Available: http://www.freepint.com/issues/060203.htm\#feature.

[4] I. J. Rotkin and K. J. Dood, A History of Patent Classification in the United States Patent and Trademark Office, Patent Documentation Society, Arlington, Virginia, 1999.

[5] U.S. Patent and Trademark Office. (2003). Examiner Handbook to the U.S. Patent Classification System [Online]. Available: http://www.uspto.gov/patents/resources/classification/handbook/index.jsp.

[6] Patent and Trademark Depository Library Program, U.S. Patent and Trademark Office. How to Conduct a Preliminary U.S. Patent Search: A Step by Step Strategy, U.S. Patent and Trademark Office [Online]. Available: http://www.uspto.gov/go/ptdl/CBT/.

[7] Penn State University Libraries. (2009). Patents Tutorial [Online]. Available: http://www.libraries.psu.edu/psul/researchguides/patents.html.

[8] University of Central Florida Libraries. (2011). Patent and Trademark Tutorials [Online], Available: http://library.ucf.edu/GovDocs/PatentsTrademarks/Tutorial.php.

[9] The Institution of Engineering and Technology. (2011). International Patent Classification in Inspec [Online]. Available: http://www.theiet.org/resources/inspec/about/records/IPC.cfm.

[10] CPC Implementation Group. (2011, Oct.). Cooperative Patent Classification (CPC): EPO and USPTO Bilateral Classification System [Online], Available: http://www.cooperativepatentclassification.org/publications/CPCPresentation.pdf.

[11] C. H. Clark, “Bicycle and the Like”, U.S. Patent 1,584,568, May 11, 1926. 